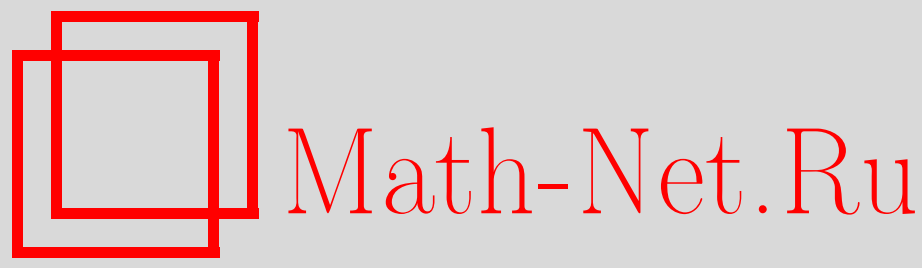

Э. Тасси, Редуцированная гамильтонова жидкостная модель для плазм с анизотропией температуры и тепловых потоков, ТМФ, 2016, том 188, номер 3, 477-496

DOI: https://doi.org/10.4213/tmf9048

Использование Общероссийского математического портала Math-Net.Ru подразумевает, что вы прочитали и согласны с пользовательским соглашением http://www . mathnet.ru/rus/agreement

Параметры загрузки:

IP : 54.197 .130 .99

26 апреля 2023 г., $12: 43: 15$

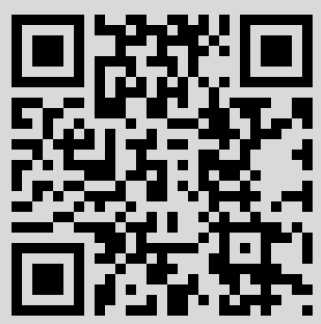




\title{
ФИЗИКА
}

Том 188, № 3

сентябрь, 2016

\section{(C) 2016 г. \\ РЕДУЦИРОВАННАЯ ГАМИЛЬТОНОВА ЖИДКОСТНАЯ МОДЕЛЬ ДЛЯ ПЛАЗМ С АНИЗОТРОПИЕЙ ТЕМПЕРАТУРЫ И ТЕПЛОВЫХ ПОТОКОВ}

\begin{abstract}
Выводится гамильтонова жидкостная модель сильно намагниченной плазмы, которая для произвольного числа типов частиц плазмы описывает эволюцию плотности, скорости и электромагнитных флуктуаций, а также флуктуаций температуры и тепловых потоков, связанную с движениями, перпендикулярными и параллельными направлению фонового магнитного поля. Модель получается как редукция бесконечной иерархии уравнений, полученных с помощью моментов кинетических функций (выраженных через полиномы Эрмита и Лагерра от скорости и магнитных моментов) гамильтоновой дрейфовой кинетической системы. Показано, что соотношение замыкания системы уравнений, непосредственно связывающее флуктуации теплового потока в направлениях, перпендикулярных и параллельных фоновому магнитному полю, позволяет получить жидкостную редукцию, которая сохраняет гамильтонов характер исходной дрейфовой кинетической модели. Найден альтернативный набор динамических переменных, в терминах которых скобка Пуассона жидкостной модели принимает простой вид непосредственной суммы скобок и позволяет без труда определить инварианты Казимира. Такие инварианты в пределе трансляционной симметрии по отношению к направлению фонового магнитного поля оказываются связанными с лагранжевыми инвариантами жидкостной модели. Указано, что связь между эволюциями параллельных и перпендикулярных тепловых потоков, получающаяся в результате замыкания системы уравнений, необходима, чтобы гарантировать существование гамильтоновой структуры со скобкой Пуассона, полученной как расширение скобки Ли-Пуассона.
\end{abstract}

Ключевые слова: жидкостная редукция, плазма, неканонические скобки Пуассона, дрейфовая кинетика.

DOI: $10.4213 / \operatorname{tmf} 9048$

Работа выполнена при финансовой поддержке CNRS (проект PEPS GEOPLASMAZ).

*Centre de Physique Théorique, Aix-Marseille Université, CNRS, Marseille, France. E-mail: tassi@cpt.univ-mrs.fr

${ }^{\dagger}$ Université de Toulon, Toulon, France 


\section{1. ВВЕДЕНИЕ}

Кинетические теории позволяют дать точное описание таких непрерывных сред, как газы и плазма. Модели среды состоят из уравнений эволюции для функций распределения, заданных на фазовом пространстве частиц; эти уравнения, возможно, связаны самосогласованным образом с уравнениями, определяющими эволюцию полей, воздействующих на динамику частиц. Хрестоматийными примерами кинетических теорий являются система Власова-Максвелла (для плазмы в присутствии электромагнитных полей) и система Власова-Пуассона (для плазмы в электростатических полях или для распределений частиц, подверженных воздействию их собственного гравитационного потенциала). Менее точный, но менее требовательный в вычислительном смысле уровень описания непрерывных сред обеспечивается жидкостными моделями, которые в эйлеровом представлении состоят из уравнений эволюции для жидкостных динамических переменных, зависящих только от пространственных координат: от плотностей частиц, моментов, температуры и т. д. Эти уравнения дополняются основными уравнениями для полей.

Динамические переменные обычно соответствуют моментам кинетических функций распределения, полученным путем взятия интеграла по пространству скоростей от функций распределения, умноженных на скорости в неотрицательных целочисленных степенях. Эти степени определяют порядок моментов. Уравнения эволюции для моментов можно затем получить непосредственно из уравнений эволюции для функций распределения. Так как уравнения эволюции для каждого момента обычно зависят также от моментов более высокого порядка, это приводит к бесконечной иерархии связанных уравнений эволюции для жидкостных переменных. Чтобы получить замкнутую систему для конечного числа жидкостных переменных, требуется процедура редукции, которую обычно называют замыканием. Замыкание предполагает, что момент данного порядка определяется моментами более низких порядков. Такие моменты более низкого порядка становятся динамическими переменными жидкостной модели, которая уже не эквивалентна первоначальному кинетическому описанию, но представляет собой систему уравнений эволюции для конечного числа динамических переменных, зависящих только от пространственных координат. Ясно, что выбор замыкания, связывающего данный момент с моментами более низких порядков, является решающим при определении характеристик жидкостной модели.

В литературе по физике плазмы можно найти немало примеров соотношений замыкания, которые удовлетворяют различным критериям. Так, в работах [1], [2] замыкание гарантирует сохранение энергии в результирующей жидкостной модели. Жидкостные модели, сохраняющие такие кинетические эффекты, как затухание Ландау, приводятся в работах [3]-[7]. Недиссипативные замыкания, которые сохраняют симметрию по отношению к обращению времени, присущую исходному кинетическому уравнению, предложены в работе [8]. Замыкание для жидкостной модели, основанное на производстве энтропии, рассмотрено в работе [9].

Несмотря на значительные усилия по нахождению замыканий для жидкостных моделей, о последствиях таких замыканий с точки зрения некоторых особенностей уравнений модели известно мало. В частности, во многих случаях неизвестно, обладает ли замыкание жидкостной модели гамильтоновой структурой в пределе, когда исходная кинетическая модель имеет гамильтонову структуру (что харак- 
терно, например, для систем Власова-Максвелла и Власова-Пуассона [10], [11]). Это должно быть так, разве только в жидкостную модель искусственно не вводится диссипация. В литературе по математической физике результаты, связанные с этой проблемой, приводились еще в работах [12], [13], где пояснялась связь между скобкой Пуассона для уравнения Власова и скобками Пуассона в терминах моментов. Связь между гамильтоновой динамикой моментов Власова и интегрируемостью обсуждалась в работе [14]. С другой стороны, гамильтонова структура иерархии Боголюбова-Борна-Грина-Кирквуда-Ивона для уравнений моментов была представлена в работе [15]. В статье [16] были заданы гамильтоновы структуры для конечных редукций уравнений кинетики жидкости, возникающие из системы Власова-Пуассона. Недавно в этом контексте из систем Власова-Пуассона были получены некоторые результаты по определению гамильтоновых замыканий в случае моделей с тремя [17] и четырьмя [18] полями, а в случае моделей с двумя полями результаты были выведены из дрейфовых кинетических [19], [20] и гирокинетических [21] систем. В работе [22] замыкание, сохраняющее гамильтонову структуру жидкостной модели и удерживающее произвольное число моментов, было выведено из дрейфовой кинетической системы.

Результаты, представленные в настоящей статье, относятся к исследованиям замыканий, которые сохраняют гамильтонову структуру исходной гамильтоновой кинетической теории. Мы рассматриваем в качестве исходной систему дрейфовых кинетических уравнений, дополненную с помощью условия квазинейтральности и закона Ампера уравнениями для электромагнитных полей. Дрейфовые кинетические уравнения (см., например, работу [23]) вызывают интерес в связи с низкочастотными явлениями в плазме в присутствии сильного магнитного поля. Действительно, при этих предположениях можно усреднить быстрое вращение частиц вокруг высокоинтенсивной компоненты магнитного поля, так как такое движение происходит на слишком малых временных масштабах по сравнению с интересующими нас частотами. В результате дрейфовое кинетическое уравнение имеет преимущество по сравнению с уравнением для функции распределения Власова, заданной на шестимерном фазовом пространстве. Это связано с тем, что дрейфовое кинетическое уравнение записывается в редуцированном пятимерном фазовом пространстве, где вращательной угловой координатой пренебрегают как несущественной при низкочастотных явлениях. Гамильтонова дрейфовая кинетическая модель, принятая в настоящей статье, может быть выведена из гирокинетической модели [2] с помощью применения некоторого числа упрощающих предположений, согласно которым мы пренебрегаем эффектами, связанными с конечным радиусом Лармора, неоднородностями фона и с диссипативными членами.

Цель статьи - показать, что существует замыкание, с помощью которого можно вывести из гамильтоновой дрейфовой кинетической системы гамильтонову жидкостную модель эволюции шести полевых переменных для каждого типа частиц. Точнее, если $\mathcal{N}$ - число типов частиц плазмы, мы можем построить $6 \mathcal{N}$-полевую гамильтонову жидкостную модель, описывающую для каждого типа частиц и самосогласованно с электромагнитными полями эволюцию плотности, канонического жидкостного импульса, флуктуаций температуры и тепловых потоков, возникающих в результате параллельных и перпендикулярных магнитному полю движений частиц. Эти полевые переменные пропорциональны моментам обобщенной функции распределения (и выражены через полиномы Эрмита и Лагерра от соответственно 
нормированной скорости вдоль сильной компоненты магнитного поля и магнитного момента).

По отношению к недавним результатам работ [19]-[21], [24] наша модель также дополнительно учитывает средние магнитного момента частицы и, таким образом, приводит к жидкостной модели, которая может описать явления, связанные с анизотропией температуры и теплового потока.

Существование гамильтоновой структуры в получающейся жидкостной модели помимо гарантии отсутствия паразитных диссипативных эффектов и возможности получить точное выражение для сохраняющейся полной энергии может также дать информацию о других законах сохранения системы. Действительно, как и в случае гамильтоновой структуры уравнений для непрерывных сред в эйлеровом описании, оказывается, что такая жидкостная модель обладает скобкой Пуассона неканонического типа (см., например, работы [25], [26]). Скобки Пуассона этого типа характеризуются наличием инвариантов Казимира, что налагает ограничения на динамику системы. Благодаря наличию гамильтоновой структуры в нашей модели мы можем найти инварианты Казимира, которые особенно важны в двумерном пределе, когда система является трансляционно-инвариантной вдоль направления высокоинтенсивной компоненты магнитного поля. В этом пределе возникают бесконечные семейства инвариантов Казимира. Инварианты Казимира можно определить благодаря замене переменных, в терминах которых скобка Пуассона для нашей модели записывается в наиболее простом виде, имея структуру непосредственной суммы скобок. Этот альтернативный набор переменных, которые мы называем нормальными полями, оказывается связанным с существованием $6 \mathcal{N}$ лагранжевых инвариантов для модели в ее двумерном пределе.

Статья построена следующим образом. В разделе 2 вводится исходная дрейфовая кинетическая модель и ее гамильтонова структура. В разделе 3 мы вводим иерархию жидкостных кинетических уравнений в терминах моментов, выраженных через полиномы Эрмита и Лагерра, и представляем соотношение замыкания. Гамильтонова структура получающейся в результате жидкостной модели обсуждается в разделе 4 , а в разделе 5 мы вводим нормальные поля и представляем инварианты Казимира. Выводы приведены в разделе 6.

\section{2. ИСХОДНАЯ ГАМИЛЬТОНОВА ДРЕЙФОВАЯ КИНЕТИЧЕСКАЯ МОДЕЛЬ}

Мы рассматриваем систему дрейфовых кинетических уравнений в декартовой системе координат $(x, y, z)$ вида

$$
\frac{\partial g_{s}}{\partial t}+\frac{c}{B}\left[\phi-\frac{v}{c} A, g_{s}\right]+v \frac{\partial}{\partial z}\left(g_{s}+q_{s} \frac{\mathcal{F}_{s}}{T_{s}}\left(\phi-\frac{v}{c} A\right)\right)=0,
$$

дополненную соотношением квазинейтральности

$$
\frac{n_{0} M c^{2}}{B^{2}} \Delta_{\perp} \phi=-\sum_{s} q_{s} \int d \mathcal{W}_{s} g_{s}
$$

и законом Ампера

$$
\frac{c}{4 \pi} \Delta_{\perp} A-\sum_{s} \frac{q_{s}^{2} n_{0}}{m_{s} c} A=-\sum_{s} q_{s} \int d \mathcal{W}_{s} v g_{s}
$$


Здесь нижний индекс $s=1, \ldots, \mathcal{N}$ отвечает одному из $\mathcal{N}$ типов частиц плазмы, и, чтобы упростить обозначения, здесь и далее мы считаем, что индекс суммирования $s$ изменяется от 1 до $\mathcal{N}$, но не пишем этого. Динамическая полевая переменная $g_{s}$ определяется как

$$
g_{s}\left(x, y, z, v, \mu_{s}, t\right)=\tilde{f}\left(x, y, z, v, \mu_{s}, t\right)+\frac{q_{s}}{T_{s}} \frac{v}{c} \mathcal{F}_{s}\left(v, \mu_{s}\right) A(x, y, z, t),
$$

где $\tilde{f}$ - возмущение функции распределения для $s$-го типа частиц относительно равновесного распределения Максвелла

$$
\mathcal{F}_{s}\left(v, \mu_{s}\right)=n_{0}\left(\frac{m_{s}}{2 \pi T_{s}}\right)^{3 / 2} \exp \left(-\frac{v^{2}}{2 v_{\mathrm{t}_{s}}^{2}}-\frac{\mu_{s} B}{m_{s} v_{\mathrm{t}_{s}}^{2}}\right) .
$$

В приведенных выше выражениях $v$ - скорость вдоль направления $z$, совпадающего с направлением магнитного поля, $\mu_{s}=m_{s}\left(v_{x}^{2}+v_{y}^{2}\right) / 2 B$ - магнитный момент частиц $s$-го типа, а константы $q_{s}$ и $m_{s}, T_{s}, v_{\mathrm{t}_{s}}=\sqrt{T_{s} / m_{s}}, c$ и $n_{0}$ суть заряд и масса частицы, равновесная температура частиц $s$-го типа, тепловая скорость, скорость света и равновесная плотность. Магнитное поле дается выражением $\mathbf{B}(x, y, z, t)=\nabla A(x, y, z, t) \times \hat{z}+B \hat{z}$, где $B$ - постоянная, а $\phi=\phi(x, y, z, t)$ обозначает электростатический потенциал. В уравнении (2) постоянная $M=\sum_{s} m_{s}$. Символом $\Delta_{\perp}$ мы обозначаем оператор Лапласа $\partial_{x x}+\partial_{y y}$ по переменным, перпендикулярным направлению $z$, а скобка $[\cdot, \cdot]$ определяется как $[f, g]=\partial_{x} f \partial_{y} g-\partial_{y} f \partial_{x} g$ для двух функций $f$ и $g$. Наконец, элемент объема $d \mathcal{W}$ есть $d \mathcal{W} \mathcal{F}_{s}=2 \pi\left(B / m_{s}\right) d \mu_{s} d v$.

Пространственные координаты $x, y$ и $z$ изменяются в трехмерном торе $\mathbb{T}^{3}$, скорость $v \in \mathbb{R}$, для всех $s$ момент $\mu_{s} \geqslant 0$ и $t \geqslant 0$. Предполагается, что возмущение $\tilde{f}$ функции распределения достаточно быстро стремится к нулю при $v \rightarrow \pm \infty$, так что при интегрировании по частям по переменной $v$ граничные члены обращаются в нуль. Также предполагается, что электростатический потенциал $\phi$ имеет нулевое среднее, поэтому оператор Лапласа в уравнении (2) обратим.

Модель (1)-(3) можно вывести из гирокинетической модели (см., например, paботу [2]) в пределе нулевого радиуса Лармора и при условии отсутствия диссипации и магнитных неоднородностей фона. Модель предполагает общую аппроксимацию с точностью $\delta f$, которая допустима для плазмы, близкой к состоянию равновесия, и со слабыми изменениями вдоль направляющего поля, т. е.

$$
\tilde{f} \ll \mathcal{F}_{s}, \quad \frac{\partial}{\partial z} \ll \frac{\partial}{\partial x}, \quad \frac{\partial}{\partial z} \ll \frac{\partial}{\partial y} .
$$

Что касается уравнения (2), видно, что по сравнению с условием квазинейтральности, принятым в работе [2], мы пренебрегли вкладом конечного радиуса Лармоpa, который зависит от температуры, отвечающей движению в перпендикулярном направлении, тогда как вклад главного порядка для поляризационного члена был сохранен. Конкретный пример, в котором это предположение оправданно, соответствует случаю, когда в плазме присутствуют два типа частиц: электроны, сохраняющие анизотропию температуры и теплового потока, и ионы, допускающие изотермическое описание.

Дрейфовая кинетическая модель (1)-(3) обладает гамильтоновой структурой, которая является непосредственным расширением гамильтоновой структуры модели, 
рассмотренной в работе [27]. Эта гамильтонова структура включает в себя гамильтонов функционал

$$
H\left(g_{1}, \ldots, g_{\mathcal{N}}\right)=\sum_{s} \frac{1}{2} \int d^{3} x d \mathcal{W}_{s}\left[T_{s} \frac{g_{s}^{2}}{\mathcal{F}_{s}}+q_{s} g_{s}\left(\phi-\frac{v}{c} A\right)\right]
$$

и неканоническую скобку Пуассона

$$
\{F, G\}=\sum_{s}\{F, G\}_{s}=-\sum_{s} \int d^{3} x d \mathcal{W}_{s}\left(\frac{c}{q_{s} B} g_{s}\left[F_{g_{s}}, G_{g_{s}}\right]+v \frac{\mathcal{F}_{s}}{T_{s}} F_{g_{s}} \frac{\partial G_{g_{s}}}{\partial z}\right)
$$

где нижние индексы функционалов $F$ и $G$ обозначают функциональные производные.

Действительно, общее выражение для гамильтоновой системы, описывающей динамику $N$ полевых переменных $\chi_{1}, \ldots, \chi_{N}$ дается уравнением

$$
\frac{\partial \chi_{i}}{\partial t}=\left\{\chi_{i}, H\right\}, \quad i=1, \ldots, N
$$

где $H=H\left(\chi_{1}, \ldots, \chi_{N}\right)$ - гамильтонов функционал и $\{\cdot, \cdot\}$ - скобка Пуассона. Положив $N=\mathcal{N}$, заменив $\chi_{1}, \ldots, \chi_{\mathcal{N}}$ на $g_{1}, \ldots, g_{\mathcal{N}}$, а $H$ на гамильтонов потенциал $(6)$ и использовав в качестве скобки Пуассона скобку (7), мы получаем в точности дрейфовое кинетическое эволюционное уравнение (1).

Заметим, что в то время как скобка Пуассона (7) есть непосредственная сумма скобок Пуассона, каждая из которых связана с определенным типом частиц, динамическая связь между частицами разных типов возникает из-за наличия в гамильтониане (6) слагаемого, содержащего $\phi$ и $A$ и поэтому зависящего от всех типов частиц согласно (2) и (3). С другой стороны, первое слагаемое в (6) возникает из разложения до второго порядка относительно равновесного распределения Максвелла (5) функционала свободной энергии в первоначальной полной (без $\delta f$-приближения) дрейфовой кинетической модели.

\section{3. УРАВНЕНИЯ ДЛЯ КИНЕТИКИ ЖИДКОСТИ}

Иерархию эволюционных уравнений для динамических переменных, зависящих только от пространственных координат $x, y, z$ и от времени, можно получить из дрейфовых кинетических уравнений (1). В качестве динамических переменных для частиц данного типа $s$ мы выбираем моменты $g_{m n_{s}}$, определенные как

$$
g_{m n_{s}}(x, y, z, t)=\frac{1}{n_{0} \sqrt{m !}} \int d \mathcal{W}_{s} H_{m}\left(\frac{v}{v_{\mathrm{t}_{s}}}\right) L_{n}\left(\frac{\mu_{s} B}{m_{s} v_{\mathrm{t}_{s}}^{2}}\right) g_{s}\left(x, y, z, v, \mu_{s}, t\right),
$$

где $H_{m}$ и $L_{n}$ - полиномы Эрмита и Лагерра неотрицательных целых порядков $m$ и $n$. Напомним, что такие полиномы могут быть определены следующим образом:

$$
H_{m}(x)=(-1)^{m} e^{x^{2} / 2} \frac{d^{m}}{d x^{m}} e^{-x^{2} / 2}, \quad L_{n}(x)=\frac{e^{x}}{n !} \frac{d^{n}}{d x^{n}}\left(x^{n} e^{-x}\right)
$$


Первые полиномы Эрмита и Лагерра, которые особенно интересны при нашем анализе, записываются так:

$$
\begin{gathered}
H_{0}\left(\frac{v}{v_{\mathrm{t}_{s}}}\right)=1, \quad H_{1}\left(\frac{v}{v_{\mathrm{t}_{s}}}\right)=\frac{v}{v_{\mathrm{t}_{s}}}, H_{2}\left(\frac{v}{v_{\mathrm{t}_{s}}}\right)=\frac{v^{2}}{v_{\mathrm{t}_{s}}^{2}}-1, \quad H_{3}\left(\frac{v}{v_{\mathrm{t}_{s}}}\right)=\frac{v^{3}}{v_{\mathrm{t}_{s}}^{3}}-3 \frac{v}{v_{\mathrm{t}_{s}}}, \\
L_{0}\left(\frac{\mu_{s} B}{m_{s} v_{\mathrm{t}_{s}}^{2}}\right)=1, \quad L_{1}\left(\frac{\mu_{s} B}{m_{s} v_{\mathrm{t}_{s}}^{2}}\right)=1-\frac{\mu_{s} B}{m_{s} v_{\mathrm{t}_{s}}^{2}} .
\end{gathered}
$$

Выбор полиномов Эрмита и Лагерра от параллельных скоростей и магнитных моментов как "весовых" функций в уравнении (9) оказывается удобным благодаря соотношению ортогональности

$$
\int d \mathcal{W}_{s} H_{m}\left(\frac{v}{v_{\mathrm{t}_{s}}}\right) H_{i}\left(\frac{v}{v_{\mathrm{t}_{s}}}\right) L_{n}\left(\frac{\mu_{s} B}{m_{s} v_{\mathrm{t}_{s}}^{2}}\right) L_{j}\left(\frac{\mu_{s} B}{m_{s} v_{\mathrm{t}_{s}}^{2}}\right) \mathcal{F}_{s}=n_{0} m ! \delta_{m i} \delta_{n j},
$$

которое содержит равновесное распределение Максвелла $\mathcal{F}_{s}$. Динамическая переменная $g_{s}$ разлагается по базису полиномов Эрмита и Лагерра следующим образом:

$$
g_{s}\left(x, y, z, v, \mu_{s}, t\right)=\sum_{m, n=0}^{+\infty} \frac{1}{\sqrt{m !}} H_{m}\left(\frac{v}{v_{\mathrm{t}_{s}}}\right) L_{n}\left(\frac{\mu_{s} B}{m_{s} v_{\mathrm{t}_{s}}^{2}}\right) \mathcal{F}_{s}\left(v, \mu_{s}\right) g_{m n_{s}}(x, y, z, t) .
$$

Отсюда следует выражение (9), если использовать соотношение (12).

Мы рассматриваем эволюцию моментов $g_{i 0_{s}}, i=0,1,2,3$, и $g_{k 1_{s}}, k=0,1$. Эти моменты пропорциональны наиболее значимым физическим динамическим переменным, обычно принятым в анизотропно редуцированных жидкостных моделях плазмы в присутствии сильного направляющего поля. Соответствие между моментами и физическими переменными дается формулами

$$
\begin{gathered}
g_{00_{s}}=\frac{n_{s}}{n_{0}}, \quad g_{10_{s}}=\frac{u_{s}}{v_{\mathrm{t}_{s}}}+q_{s} \frac{A}{m_{s} v_{\mathrm{t}_{s}} c}, \quad g_{20_{s}}=\frac{1}{\sqrt{2}} \frac{T_{\|_{s}}}{T_{s}}, \quad g_{30 s}=\sqrt{\frac{2}{3}} \frac{1}{n_{0}} \frac{q_{\|_{s}}}{T_{s} v_{\mathrm{t}_{s}}}, \\
g_{01_{s}}=-\frac{T_{\perp s}}{T_{s}}, \quad g_{11_{s}}=-\frac{1}{n_{0}} \frac{q_{\perp s}}{T_{s} v_{\mathrm{t}_{s}}},
\end{gathered}
$$

где для каждого типа $s=1, \ldots, \mathcal{N}$

$$
n_{s}=\int d \mathcal{W}_{s} \tilde{f}_{s}, \quad u_{s}=\frac{1}{n_{0}} \int d \mathcal{W}_{s} v \tilde{f}_{s}
$$

суть флуктуации плотности и скорости,

$$
T_{\|_{s}}=\frac{1}{n_{0}} \int d \mathcal{W}_{s}\left(m_{s} v^{2}-T_{s}\right) \tilde{f}_{s}, \quad T_{\perp_{s}}=\frac{1}{n_{0}} \int d \mathcal{W}_{s}\left(\mu_{s} B-T_{s}\right) \tilde{f}_{s}
$$

суть флуктуации температур, отвечающих параллельному и перпендикулярному движению, тогда как

$$
q_{\|_{s}}=\frac{1}{2} \int d \mathcal{W}_{s}\left(m_{s} v^{2}-3 T_{s}\right) v \tilde{f}_{s}, \quad q_{\perp s}=\int d \mathcal{W}_{s}\left(\mu_{s} B-T_{s}\right) v \tilde{f}_{s}
$$

представляют собой флуктуации перпендикулярного и параллельного тепловых потоков. 
Умножим обе части уравнения (1) на $H_{m}\left(v / v_{\mathrm{t}_{s}}\right) L_{n}\left(\mu_{s} B / m_{s} v_{\mathrm{t}_{s}}^{2}\right)$ и проинтегрируем по $d \mathcal{W}_{s}$, тогда для шести моментов (14) получим следующую систему эволюционных уравнений:

$$
\begin{aligned}
\frac{\partial g_{00_{s}}}{\partial t}= & -\frac{c}{B}\left[\phi, g_{00_{s}}\right]+\frac{v_{\mathrm{t}_{s}}}{B}\left[A, g_{10_{s}}\right]-v_{\mathrm{t}_{s}} \frac{\partial g_{10_{s}}}{\partial z}+v_{\mathrm{t}_{s}}^{2} \frac{q_{s}}{c T_{s}} \frac{\partial A}{\partial z} \\
\frac{\partial g_{10_{s}}}{\partial t}= & -\frac{c}{B}\left[\phi, g_{10_{s}}\right]+\sqrt{2} \frac{v_{\mathrm{t}_{s}}}{B}\left[A, g_{20_{s}}\right]+\frac{v_{\mathrm{t}_{s}}}{B}\left[A, g_{00_{s}}\right]- \\
& -\sqrt{2} v_{\mathrm{t}_{s}} \frac{\partial g_{20_{s}}}{\partial z}-v_{\mathrm{t}_{s}} \frac{\partial g_{00_{s}}}{\partial z}-v_{\mathrm{t}_{s}} \frac{q_{s}}{T_{s}} \frac{\partial \phi}{\partial z} \\
\frac{\partial g_{20_{s}}}{\partial t}= & -\frac{c}{B}\left[\phi, g_{20_{s}}\right]+\sqrt{3} \frac{v_{\mathrm{t}_{s}}}{B}\left[A, g_{30_{s}}\right]+\sqrt{2} \frac{v_{\mathrm{t}_{s}}}{B}\left[A, g_{10_{s}}\right]- \\
& -\sqrt{3} v_{\mathrm{t}_{s}} \frac{\partial g_{30_{s}}}{\partial z}-\sqrt{2} v_{\mathrm{t}_{s}} \frac{\partial g_{10_{s}}}{\partial z}+\sqrt{2} v_{\mathrm{t}_{s}}^{2} \frac{q_{s}}{c T_{s}} \frac{\partial A}{\partial z} \\
\frac{\partial g_{30_{s}}}{\partial t}= & -\frac{c}{B}\left[\phi, g_{30_{s}}\right]+2 \frac{v_{\mathrm{t}_{s}}}{B}\left[A, g_{40_{s}}\right]+\sqrt{3} \frac{v_{\mathrm{t}_{s}}}{B}\left[A, g_{20}\right]- \\
& -2 v_{\mathrm{t}_{s}} \frac{\partial g_{40_{s}}}{\partial z}-\sqrt{3} v_{\mathrm{t}_{s}} \frac{\partial g_{20_{s}}}{\partial z}, \\
\frac{\partial g_{01_{s}}}{\partial t}= & -\frac{c}{B}\left[\phi, g_{01_{s}}\right]+\frac{v_{\mathrm{t}_{s}}}{B}\left[A, g_{11_{s}}\right]-v_{\mathrm{t}_{s}} \frac{\partial g_{11_{s}}}{\partial z}, \\
\frac{\partial g_{11_{s}}}{\partial t}= & -\frac{c}{B}\left[\phi, g_{11_{s}}\right]+\sqrt{2} \frac{v_{\mathrm{t}_{s}}\left[A, g_{21_{s}}\right]+\frac{v_{\mathrm{t}_{s}}}{B}\left[A, g_{01_{s}}\right]-}{}-\sqrt{2} v_{\mathrm{t}_{s}} \frac{\partial g_{21_{s}}}{\partial z}-v_{\mathrm{t}_{s}} \frac{\partial g_{01_{s}}}{\partial z},
\end{aligned}
$$

где было использовано рекуррентное соотношение $H_{m+1}(x)=x H_{m}(x)-m H_{m-1}(x)$.

Из уравнений (18) и (20) следует, что система не замкнута, так как эти два уравнения зависят от неопределенных моментов $g_{40 s}$ и $g_{21_{s}}$. Это приводит к проблеме замыкания системы: требуется выразить $g_{40}$ и $g_{21_{s}}$ через шесть моментов более низких порядков. Напомним, что нам необходимо сохранить в жидкостной модели гамильтонов характер исходной дрейфовой кинетической модели. Далее мы покажем, что замыкание системы (15)-(20) с помощью соотношений

$$
g_{40 s}=\alpha_{s} g_{11_{s}}, \quad g_{21_{s}}=\sqrt{2} \alpha_{s} g_{30_{s}}
$$

с ненулевой константой $\alpha_{s}$ ведет к жидкостной модели с гамильтоновой структурой.

\section{4. ГАМИЛЬТОНОВА АНИЗОТРОПНАЯ ЖИДКОСТНАЯ МОДЕЛЬ}

Чтобы упростить обозначения, мы переименуем моменты следующим образом:

$$
\begin{array}{lll}
g_{0_{s}}=g_{00_{s}}, & g_{1_{s}}=g_{10_{s}}, & g_{2_{s}}=g_{20_{s}}, \\
g_{3_{s}}=g_{30_{s}}, & g_{4_{s}}=g_{01_{s}}, & g_{5_{s}}=g_{11_{s}} .
\end{array}
$$

Применяя соотношение замыкания (21), мы получаем анизотропную жидкостную модель

$$
\frac{\partial g_{0_{s}}}{\partial t}=-\frac{c}{B}\left[\phi, g_{0_{s}}\right]+\frac{v_{\mathrm{t}_{s}}}{B}\left[A, g_{1_{s}}\right]-v_{\mathrm{t}_{s}} \frac{\partial g_{1_{s}}}{\partial z}+v_{\mathrm{t}_{s}}^{2} \frac{q_{s}}{c T_{s}} \frac{\partial A}{\partial z},
$$




$$
\begin{aligned}
\frac{\partial g_{1_{s}}}{\partial t}= & -\frac{c}{B}\left[\phi, g_{1_{s}}\right]+\sqrt{2} \frac{v_{\mathrm{t}_{s}}}{B}\left[A, g_{2_{s}}\right]+\frac{v_{\mathrm{t}_{s}}}{B}\left[A, g_{0_{s}}\right]- \\
& -\sqrt{2} v_{\mathrm{t}_{s}} \frac{\partial g_{2_{s}}}{\partial z}-v_{\mathrm{t}_{s}} \frac{\partial g_{0_{s}}}{\partial z}-v_{\mathrm{t}_{s}} \frac{q_{s}}{T_{s}} \frac{\partial \phi}{\partial z} \\
\frac{\partial g_{2_{s}}}{\partial t}= & -\frac{c}{B}\left[\phi, g_{2_{s}}\right]+\sqrt{3} \frac{v_{\mathrm{t}_{s}}}{B}\left[A, g_{3_{s}}\right]+\sqrt{2} \frac{v_{\mathrm{t}_{s}}}{B}\left[A, g_{1_{s}}\right]- \\
& -\sqrt{3} v_{\mathrm{t}_{s}} \frac{\partial g_{3_{s}}}{\partial z}-\sqrt{2} v_{\mathrm{t}_{s}} \frac{\partial g_{1_{s}}}{\partial z}+\sqrt{2} v_{\mathrm{t}_{s}}^{2} \frac{q_{s}}{c T_{s}} \frac{\partial A}{\partial z}, \\
\frac{\partial g_{3_{s}}}{\partial t}= & -\frac{c}{B}\left[\phi, g_{3_{s}}\right]+2 \alpha_{s} \frac{v_{\mathrm{t}_{s}}}{B}\left[A, g_{5_{s}}\right]+\sqrt{3} \frac{v_{\mathrm{t}_{s}}}{B}\left[A, g_{2_{s}}\right]- \\
& -2 \alpha_{s} v_{\mathrm{t}_{s}} \frac{\partial g_{5_{s}}}{\partial z}-\sqrt{3} v_{\mathrm{t}_{s}} \frac{\partial g_{2_{s}}}{\partial z}, \\
\frac{\partial g_{4_{s}}}{\partial t}= & -\frac{c}{B}\left[\phi, g_{4_{s}}\right]+\frac{v_{\mathrm{t}_{s}}}{B}\left[A, g_{5_{s}}\right]-v_{\mathrm{t}_{s}} \frac{\partial g_{5_{s}}}{\partial z}, \\
\frac{\partial g_{5_{s}}}{\partial t}= & -\frac{c}{B}\left[\phi, g_{5_{s}}\right]+2 \alpha_{s} \frac{v_{\mathrm{t}_{s}}}{B}\left[A, g_{3_{s}}\right]+\frac{v_{\mathrm{t}_{s}}}{B}\left[A, g_{4_{s}}\right]- \\
& -2 \alpha_{s} v_{\mathrm{t}_{s}} \frac{\partial g_{3_{s}}}{\partial z}-v_{\mathrm{t}_{s}} \frac{\partial g_{4_{s}}}{\partial z} .
\end{aligned}
$$

Чтобы показать, что данная система обладает гамильтоновой структурой, нам необходимо записать ее в виде (8). Естественный выбор для $6 \mathcal{N}$ полевых переменных задается равенствами $\chi_{i_{s}}=g_{i_{s}}, i=0,1, \ldots, 5$.

Естественный кандидат на роль гамильтонова функционала получается непосредственно из выражения (6) для гамильтониана порождающей модели с помощью замены $g_{s}$ на усеченную версию ряда (13):

$$
\begin{aligned}
& g_{s}\left(x, y, z, v, \mu_{s}, t\right)=\mathcal{F}_{s}\left(v, \mu_{s}\right)\left[g_{0 s}(x, y, z, t) H_{0}\left(\frac{v}{v_{\mathrm{t}_{s}}}\right) L_{0}\left(\frac{\mu_{s} B}{m_{s} v_{\mathrm{t}_{s}}^{2}}\right)+\right. \\
& \quad+g_{1_{s}}(x, y, z, t) H_{1}\left(\frac{v}{v_{\mathrm{t}_{s}}}\right) L_{0}\left(\frac{\mu_{s} B}{m_{s} v_{\mathrm{t}_{s}}^{2}}\right)+\frac{1}{\sqrt{2}} g_{2_{s}}(x, y, z, t) H_{2}\left(\frac{v}{v_{\mathrm{t}_{s}}}\right) L_{0}\left(\frac{\mu_{s} B}{m_{s} v_{\mathrm{t}_{s}}^{2}}\right)+ \\
& \quad+\frac{1}{\sqrt{6}} g_{3 s}(x, y, z, t) H_{3}\left(\frac{v}{v_{\mathrm{t}_{s}}}\right) L_{0}\left(\frac{\mu_{s} B}{m_{s} v_{\mathrm{t}_{s}}^{2}}\right)+g_{4_{s}}(x, y, z, t) H_{0}\left(\frac{v}{v_{\mathrm{t}_{s}}}\right) L_{1}\left(\frac{\mu_{s} B}{m_{s} v_{\mathrm{t}_{s}}^{2}}\right)+ \\
& \left.\quad+g_{5_{s}}(x, y, z, t) H_{1}\left(\frac{v}{v_{\mathrm{t}_{s}}}\right) L_{1}\left(\frac{\mu_{s} B}{m_{s} v_{\mathrm{t}_{s}}^{2}}\right)\right]
\end{aligned}
$$

где сохраняется лишь вклад от моментов, включенных в модель. Подставляя усеченный ряд (28) в дрейфовый кинетический гамильтониан (6), мы получаем с помощью соотношения ортогональности (12) выражение

$$
H\left(g_{01}, \ldots, g_{5 \mathcal{N}}\right)=\frac{1}{2} \sum_{s} \int d^{3} x\left[n_{0} T_{s} \sum_{i=0}^{5} g_{i_{s}}^{2}+q_{s} n_{0} g_{0_{s}} \phi-q_{s} n_{0} \frac{v_{\mathrm{t}_{s}}}{c} g_{1_{s}} A\right]
$$

Этот функционал является сохраняющейся величиной для системы (22)-(27) и, следовательно, может рассматриваться как кандидат на роль гамильтониана. В надлежащем пределе он отвечает функционалу полной сохраняющейся энергии гирожидкостной модели из работы [2]. 
Что касается скобки Пуассона, то на основе уравнений модели можно предположить, что она должна иметь вид $\{F, G\}=\sum_{s}\{F, G\}_{s}$, где

$$
\{F, G\}_{s}=-\frac{c}{q_{s} B n_{0}} \sum_{i, j, k=0}^{5}\left(W_{k}^{i j}\right)_{s} \int d^{3} x g_{k_{s}}\left[F_{i_{s}}, G_{j_{s}}\right]-\frac{v_{\mathrm{t}_{s}}}{n_{0} T_{s}} \sum_{i, j=0}^{5}\left(\mathcal{A}^{i j}\right)_{s} \int d^{3} x F_{i s} \frac{\partial G_{j_{s}}}{\partial z} .
$$

В выражении $(30)\left(W_{k}^{i j}\right)_{s}$ и $\left(\mathcal{A}^{i j}\right)_{s}$ являются константами, нижние индексы функционалов обозначают функциональные производные: например, $F_{i_{s}}=\delta F / \delta g_{i_{s}}$. Скобки Пуассона вида (30) очень часто встречаются в редуцированных гамильтоновых жидкостных моделях плазмы при наличии сильного магнитного поля. Примеры таких моделей можно найти в работах [28]-[36].

Чтобы определить коэффициенты $\left(W_{k}^{i j}\right)_{s}$ и $\left(\mathcal{A}^{i j}\right)_{s}$, задающие скобку Пуассона, мы поступаем следующим образом. Сначала рассмотрим случай, когда $\left(\mathcal{A}^{i j}\right)_{s}=0$ для всех $i, j=0,1, \ldots, 5$, и будем искать такие коэффициенты $\left(W_{k}^{i j}\right)_{s}$, чтобы операция

$$
\{F, G\}_{\perp_{s}}=-\frac{c}{q_{s} B n_{0}} \sum_{i, j, k=0}^{5}\left(W_{k}^{i j}\right)_{s} \int d^{3} x g_{k_{s}}\left[F_{i s}, G_{j_{s}}\right]
$$

сама по себе являлась скобкой Пуассона и чтобы в комбинации с претендентом (29) на роль гамильтониана она давала уравнения (22)-(27) в "перпендикулярном" пределе, т. е. когда мы пренебрегаем всеми членами с производными по z. Далее, опираясь на методику из работы [29], мы зададим коэффициенты $\left(\mathcal{A}^{i j}\right)_{s}$ так, чтобы скобка (30) в комбинации с функционалом (29) дала все уравнения модели, и убедимся в том, что результирующая скобка является скобкой Пуассона.

Для выполнения первой части процедуры мы вспомним [37], что билинейная операция (31) является скобкой Пуассона, если и только если матрицы $\mathcal{W}_{s}^{(j)}$ с элементами

$$
\left(\mathcal{W}_{s}^{(j)}\right)^{k i}=\left(W_{k}^{i j}\right)_{s}, \quad i, j, k=0,1, \ldots, 5
$$

(индекс $k$ отвечает номеру строки, индекс $i$ - номеру столбца), являются симметричными по верхним индексам, $\left(\mathcal{W}_{s}^{(j)}\right)^{k i}=\left(\mathcal{W}_{s}^{(j)}\right)^{i k}$, и попарно коммутируют. Эти два свойства в действительности эквивалентны соответственно условию антисимметричности и тождеству Якоби для скобки (31). Заметим, что тождество Лейбница для скобки (31) автоматически удовлетворяется. В дополнение к ограничениям, связанным со свойствами скобки Пуассона, мы потребуем, чтобы коэффициенты $\left(W_{k}^{i j}\right)_{s}$ (или, что эквивалентно, матрицы $\mathcal{W}_{s}^{(j)}$ ) гарантировали (если взять $H$, заданный в $(29))$ соответствие гамильтоновых эволюционных уравнений

$$
\frac{\partial g_{l_{s}}}{\partial t}=\left\{g_{l_{s}}, H\right\}_{\perp_{s}}=-\frac{c}{q_{s} B n_{0}} \sum_{j, k=0}^{5}\left(W_{k}^{l j}\right)_{s}\left[H_{j_{s}}, g_{k_{s}}\right], \quad l=0,1, \ldots, 5
$$

уравнениям (22)-(27) в пределе, когда члены с производными по $z$ равны нулю. Для этого полезно заметить, что функциональные производные гамильтониана (29) задаются выражениями

$$
\begin{gathered}
H_{0 s}=n_{0} T_{s} g_{0_{s}}+q_{s} n_{0} \phi, \quad H_{1 s}=n_{0} T_{s} g_{1_{s}}-q_{s} n_{0} \frac{v_{\mathrm{t}_{s}}}{c} A, \\
H_{i s}=n_{0} T_{s} g_{i_{s}}, \quad i=2, \ldots, 5 .
\end{gathered}
$$


На основе этих рассуждений получаем следующее. Чтобы уравнения (33) были согласованы с уравнениями (22)-(27) и чтобы скобка (31) была антисимметрична, матрицы $\mathcal{W}_{s}^{(j)}$ должны иметь вид

$$
\begin{aligned}
& \mathcal{W}_{s}^{(0)}=\left(\begin{array}{cccccc}
1 & 0 & 0 & 0 & 0 & 0 \\
0 & 1 & 0 & 0 & 0 & 0 \\
0 & 0 & 1 & 0 & 0 & 0 \\
0 & 0 & 0 & 1 & 0 & 0 \\
0 & 0 & 0 & 0 & 1 & 0 \\
0 & 0 & 0 & 0 & 0 & 1
\end{array}\right), \mathcal{W}_{s}^{(1)}=\left(\begin{array}{cccccc}
0 & 1 & 0 & 0 & 0 & 0 \\
1 & 0 & \sqrt{2} & 0 & 0 & 0 \\
0 & \sqrt{2} & 0 & \sqrt{3} & 0 & 0 \\
0 & 0 & \sqrt{3} & 0 & 0 & 2 \alpha_{s} \\
0 & 0 & 0 & 0 & 0 & 1 \\
0 & 0 & 0 & 2 \alpha_{s} & 1 & 0
\end{array}\right), \\
& \mathcal{W}_{s}^{(2)}=\left(\begin{array}{cccccc}
0 & 0 & 1 & 0 & 0 & 0 \\
0 & \sqrt{2} & 0 & \sqrt{3} & 0 & 0 \\
1 & 0 & a_{1 s} & b_{1 s} & b_{2 s} & b_{3 s} \\
0 & \sqrt{3} & b_{1 s} & b_{4 s} & c_{1 s} & c_{2 s} \\
0 & 0 & b_{2 s} & c_{1 s} & b_{7 s} & c_{3 s} \\
0 & 0 & b_{3 s} & c_{2 s} & c_{3 s} & b_{10 s}
\end{array}\right), \mathcal{W}_{s}^{(3)}=\left(\begin{array}{cccccccc}
0 & 0 & 0 & 1 & 0 & 0 \\
0 & 0 & \sqrt{3} & 0 & 0 & 2 \alpha_{s} \\
0 & \sqrt{3} & b_{1 s} & b_{4 s} & c_{1 s} & c_{2 s} \\
1 & 0 & b_{4 s} & a_{2 s} & b_{5 s} & b_{6 s} \\
0 & 0 & c_{1 s} & b_{5 s} & b_{8 s} & c_{4 s} \\
0 & 2 \alpha_{s} & c_{2 s} & b_{6 s} & c_{4 s} & b_{11 s}
\end{array}\right), \\
& \mathcal{W}_{s}^{(4)}= \\
&\left.\begin{array}{cccccccccc}
0 & 0 & 0 & 0 & 1 & 0 \\
0 & 0 & 0 & 0 & 0 & 1 \\
0 & 0 & b_{2 s} & c_{1 s} & b_{7 s} & c_{3 s} \\
0 & 0 & c_{1 s} & b_{5 s} & b_{8 s} & c_{4 s} \\
1 & 0 & b_{7 s} & b_{8 s} & a_{3 s} & b_{9 s} \\
0 & 1 & c_{3 s} & c_{4 s} & b_{9 s} & b_{12 s}
\end{array}\right), \quad \mathcal{W}_{s}^{(5)}=\left(\begin{array}{ccccccc}
0 & 0 & 0 & 0 & 0 & 1 \\
0 & 0 & 0 & 2 \alpha_{s} & 1 & 0 \\
0 & 0 & b_{3 s} & c_{2 s} & c_{3 s} & b_{10 s} \\
0 & 2 \alpha_{s} & c_{2 s} & b_{6 s} & c_{4 s} & b_{11 s} \\
0 & 1 & c_{3 s} & c_{4 s} & b_{9 s} & b_{12 s} \\
1 & 0 & b_{10 s} & b_{11 s} & b_{12 s} & a_{4 s}
\end{array}\right),
\end{aligned}
$$

где $a_{1 s}, \ldots, a_{4 s}, b_{1 s}, \ldots, b_{12 s}, c_{1 s}, \ldots, c_{4 s}$ - константы, которые определяются ограничением, связанным с тождеством Якоби.

Действительно, видно, что существуют три типа вкладов, которые следует добавить к скобке (31), чтобы не нарушалось свойство антисиммметричности и чтобы, если мы примем (29) в качестве гамильтониана, не изменялись уравнения движения. Эти три типа членов соответствуют величинам

$$
\begin{array}{lc}
\int d^{3} x g_{i_{s}}\left[F_{i_{s}}, G_{i_{s}}\right], & i=2, \ldots, 5, \\
\int d^{3} x\left[g_{i_{s}}\left[F_{j_{s}}, G_{j_{s}}\right]+g_{j_{s}}\left(\left[F_{i_{s}}, G_{j_{s}}\right]+\left[F_{j_{s}}, G_{i s}\right]\right)\right], & i, j=2, \ldots, 5, \quad i \neq j, \\
\int d^{3} x\left[g_{i_{s}}\left(\left[F_{j_{s}}, G_{k_{s}}\right]+\left[F_{k_{s}}, G_{j_{s}}\right]\right)+g_{j_{s}}\left(\left[F_{k_{s}}, G_{i_{s}}\right]+\left[F_{i_{s}}, G_{k_{s}}\right]\right)+\right. \\
\left.\quad+g_{k_{s}}\left(\left[F_{i_{s}}, G_{j_{s}}\right]+\left[F_{j_{s}}, G_{i_{s}}\right]\right)\right], \quad i, j, k=2, \ldots, 5, \quad i \neq j \neq k .
\end{array}
$$

Так как $H_{i s}=n_{0} T_{s} g_{i_{s}}$ для $i=2, \ldots, 5$, нетрудно видеть, что любая линейная комбинация членов типа (36)-(38), будучи включенной в скобку, не подходит для уравнений движения $(33)$, если $\left[g_{i_{s}}, g_{i_{s}}\right]=0$. Однако такие члены, хотя и очевидные с точки зрения динамики модели, могут оказаться существенными для выполнения тождества Якоби.

Матрицы (35) определяют общие линейные комбинации слагаемых типа (36)-(38). В частности, $a_{1 s}, \ldots, a_{4 s}$ являются коэффициентами при членах типа $(36)$, в то время как $b_{1 s}, \ldots, b_{12 s}$ и $c_{1 s}, \ldots, c_{4 s}$ являются коэффициентами при членах типа (37) и (38) соответственно. 
Как было отмечено выше, тождество Якоби для скобки (31) удовлетворяется, если и только если все матрицы (35) попарно коммутируют. Наложение этого условия ограничивает произвольные коэффициенты следующим образом:

$$
\begin{aligned}
& a_{1 s}=2 \sqrt{2}, \quad a_{2 s}=0, \quad a_{3 s}=0, \quad a_{4 s}=-\frac{1+2 \alpha_{s}^{2}-8 \alpha_{s}^{4}}{\sqrt{6} \alpha_{s}}, \\
& b_{1 s}=0, \quad b_{2 s}=0, \quad b_{3 s}=\sqrt{6} \alpha_{s}, b_{4 s}=\sqrt{2}\left(1+2 \alpha_{s}^{2}\right), \\
& b_{5 s}=0, \quad b_{6 s}=\sqrt{\frac{2}{3}} \alpha_{s}\left(1+4 \alpha_{s}^{2}\right), b_{7 s}=0, \quad b_{8 s}=0 \\
& b_{9 s}=-\frac{1-2 \alpha_{s}^{2}}{\sqrt{6} \alpha_{s}}, b_{10 s}=2 \sqrt{2} \alpha_{s}^{2}, \quad b_{11 s}=0, \quad b_{12 s}=0, \\
& c_{1 s}=\sqrt{2} \alpha_{s}, \quad c_{2 s}=0, \quad c_{3 s}=0, \quad c_{4 s}=-\sqrt{\frac{2}{3}}\left(1-2 \alpha_{s}^{2}\right) .
\end{aligned}
$$

Заметим, что решение этой системы является единственным.

Таким образом, мы показали, что система (22)-(27) в пределе, когда мы пренебрегаем членами с производными по $z$, обладает гамильтоновой структурой. Эта структура состоит из гамильтонова функционала (29) и скобки Пуассона вида (31), где $\left(\mathcal{A}^{i j}\right)_{s}=0$ при всех $i, j=0,1, \ldots, 5$ и все коэффициенты $\left(W_{k}^{i j}\right)_{s}=0$, кроме

$$
\begin{aligned}
& \left(W_{k}^{i 0}\right)_{s}=\left(W_{k}^{0 i}\right)_{s}=\delta_{k}^{i}, \quad i=0,1, \ldots, 5, \\
& \left(W_{0}^{11}\right)_{s}=1, \quad\left(W_{1}^{21}\right)_{s}=\left(W_{1}^{12}\right)_{s}=\sqrt{2}, \\
& \left(W_{2}^{11}\right)_{s}=\sqrt{2}, \quad\left(W_{2}^{31}\right)_{s}=\left(W_{2}^{13}\right)_{s}=\sqrt{3}, \\
& \left(W_{3}^{21}\right)_{s}=\left(W_{3}^{12}\right)_{s}=\sqrt{3}, \quad\left(W_{3}^{51}\right)_{s}=\left(W_{3}^{15}\right)_{s}=2 \alpha_{s}, \\
& \left(W_{4}^{51}\right)_{s}=\left(W_{4}^{15}\right)_{s}=1, \quad\left(W_{5}^{31}\right)_{s}=\left(W_{5}^{13}\right)_{s}=2 \alpha_{s}, \quad\left(W_{5}^{41}\right)_{s}=\left(W_{5}^{14}\right)_{s}=1, \\
& \left(W_{0}^{22}\right)_{s}=1, \quad\left(W_{1}^{23}\right)_{s}=\left(W_{1}^{32}\right)_{s}=\sqrt{3}, \\
& \left(W_{2}^{22}\right)_{s}=2 \sqrt{2}, \quad\left(W_{2}^{25}\right)_{s}=\left(W_{2}^{52}\right)_{s}=\sqrt{6} \alpha_{s}, \\
& \left(W_{3}^{23}\right)_{s}=\left(W_{3}^{32}\right)_{s}=\sqrt{2}\left(1+2 \alpha_{s}^{2}\right), \quad\left(W_{3}^{24}\right)_{s}=\left(W_{3}^{42}\right)_{s}=\sqrt{2} \alpha_{s}, \\
& \left(W_{4}^{23}\right)_{s}=\left(W_{4}^{32}\right)_{s}=\sqrt{2} \alpha_{s}, \quad\left(W_{5}^{22}\right)_{s}=\sqrt{6} \alpha_{s}, \quad\left(W_{5}^{25}\right)_{s}=\left(W_{5}^{52}\right)_{s}=2 \sqrt{2} \alpha_{s}^{2}, \\
& \left(W_{0}^{33}\right)_{s}=1, \quad\left(W_{1}^{35}\right)_{s}=\left(W_{1}^{53}\right)_{s}=2 \alpha_{s}, \\
& \left(W_{2}^{33}\right)_{s}=\sqrt{2}\left(1+2 \alpha_{s}^{2}\right), \quad\left(W_{2}^{34}\right)_{s}=\left(W_{2}^{43}\right)_{s}=\sqrt{2} \alpha_{s} \\
& \left(W_{3}^{35}\right)_{s}=\left(W_{3}^{53}\right)_{s}=\sqrt{\frac{2}{3}} \alpha_{s}\left(1+4 \alpha_{s}^{2}\right), \quad\left(W_{4}^{35}\right)_{s}=\left(W_{4}^{53}\right)_{s}=-\sqrt{\frac{2}{3}}\left(1-2 \alpha_{s}^{2}\right), \\
& \left(W_{5}^{33}\right)_{s}=\sqrt{\frac{2}{3}} \alpha_{s}\left(1+4 \alpha_{s}^{2}\right), \quad\left(W_{5}^{34}\right)_{s}=\left(W_{5}^{43}\right)_{s}=-\sqrt{\frac{2}{3}}\left(1-2 \alpha_{s}^{2}\right), \\
& \left(W_{0}^{44}\right)_{s}=1, \quad\left(W_{1}^{45}\right)_{s}=\left(W_{1}^{54}\right)_{s}=1, \quad\left(W_{3}^{45}\right)_{s}=\left(W_{3}^{54}\right)_{s}=-\sqrt{\frac{2}{3}}\left(1-2 \alpha_{s}^{2}\right), \\
& \left(W_{4}^{45}\right)_{s}=\left(W_{4}^{54}\right)_{s}=-\frac{1-2 \alpha_{s}^{2}}{\sqrt{6} \alpha_{s}}, \quad\left(W_{5}^{44}\right)_{s}=-\frac{1-2 \alpha_{s}^{2}}{\sqrt{6} \alpha_{s}} \\
& \left(W_{0}^{55}\right)_{s}=1, \quad\left(W_{2}^{55}\right)_{s}=2 \sqrt{2} \alpha_{s}^{2}, \quad\left(W_{5}^{55}\right)_{s}=-\frac{1+2 \alpha_{s}^{2}-8 \alpha_{s}^{4}}{\sqrt{6} \alpha_{s}} .
\end{aligned}
$$


Определим гамильтонову структуру для полной модели (22)-(27) (включающей члены с производными по z), следуя методике работы [29]. Гамильтонов функционал для полной модели соответствует гамильтониану в случае перпендикулярной редукции, т. е. функционалу (29). С другой стороны, скобка Пуассона для полной модели имеет вид (30) с коэффициентами $\left(W_{k}^{i j}\right)_{s}$, равными коэффициентам, найденным для перпендикулярной редукции, и с такими коэффициентами $\left(\mathcal{A}^{i j}\right)_{s}$, что полные уравнения модели восстанавливаются как уравнения движения, а свойства скобки Пуассона не нарушаются. Билинейность и тождество Лейбница, очевидно, удовлетворяются для любого выбора коэффициентов $\left(\mathcal{A}^{i j}\right)_{s}$. При этом выполнено условие $\left(\mathcal{A}^{i j}\right)_{s}=\left(\mathcal{A}^{j i}\right)_{s}$, в то время, как из тождества Якоби следует, что коэффициенты $\left(W_{k}^{i j}\right)_{s}$ и $\left(\mathcal{A}^{i j}\right)_{s}$ должны удовлетворять уравнениям [29]

$$
\left(W_{r}^{j k}\right)_{s}\left(\mathcal{A}^{r i}\right)_{s}=\left(W_{r}^{k i}\right)_{s}\left(\mathcal{A}^{r j}\right)_{s}=\left(W_{r}^{i j}\right)_{s}\left(\mathcal{A}^{r k}\right)_{s}, \quad i, j, k=0,1, \ldots, 5
$$

(здесь и далее подразумевается суммирование по повторяющемуся индексу $r$ ).

Из соотношений (34) получаем, что уравнения для полной модели (22)-(27) можно вывести из гамильтониана (29) и из скобки вида (30), если

$$
\left(\mathcal{A}^{i j}\right)_{s}=\left(\mathcal{W}_{s}^{(1)}\right)^{i j}
$$

где матрица $\mathcal{W}_{s}^{(1)}$ задана в $(35)$. Она является симметричной, что гарантирует антисимметричность скобки Пуассона.

Что касается тождества Якоби, заметим, что для любого фиксированного $i$ мы имеем

$$
\left(W_{r}^{k i}\right)_{s}\left(\mathcal{A}^{r j}\right)_{s}=\left(\mathcal{W}_{s}^{(i)}\right)^{r k}\left(\mathcal{W}_{s}^{(1)}\right)^{j r}=\left(\mathcal{W}_{s}^{(i)}\right)^{r j}\left(\mathcal{W}_{s}^{(1)}\right)^{k r}=\left(W_{r}^{i j}\right)_{s}\left(\mathcal{A}^{r k}\right)_{s},
$$

и это дает второе равенство в (41). При получении второго равенства в (43) мы использовали тот факт, что матрица $\left(\mathcal{W}_{s}^{(i)}\right)^{r k}\left(\mathcal{W}_{s}^{(1)}\right)^{j r}$ симметрична, если все матрицы $W_{s}^{(j)}$ являются симметричными и попарно коммутируют. Аналогично имеем

$$
\left(W_{r}^{i j}\right)_{s}\left(\mathcal{A}^{r k}\right)_{s}=\left(\mathcal{W}_{s}^{(j)}\right)^{r i}\left(\mathcal{W}_{s}^{(1)}\right)^{k r}=\left(\mathcal{W}_{s}^{(j)}\right)^{r k}\left(\mathcal{W}_{s}^{(1)}\right)^{i r}=\left(W_{r}^{j k}\right)_{s}\left(\mathcal{A}^{r i}\right)_{s},
$$

что доказывает оставшееся равенство в (41). Таким образом, тождество Якоби удовлетворяется.

Итак, мы показали, что модель (22)-(27) допускает гамильтонову структуру. Гамильтонов функционал задается выражением (29). Скобка Пуассона определяется формулой (30) с коэффициентами $\left(W_{k}^{i j}\right)_{s}$, которые все равны нулю, кроме тех, что выписаны в $(40)$, и с коэффициентами $\left(\mathcal{A}^{i j}\right)_{s}$, заданными уравнениями $(42)$.

В этом месте в целях наглядности (и чтобы улучшить согласие с существующей литературой) нам представляется полезным переформулировать уравнения модели (22)-(27) в терминах размерных физических переменных, введенных в (14). Тогда модель может быть записана как

$$
\begin{aligned}
\frac{\partial n_{s}}{\partial t} & =-\frac{c}{B}\left[\phi, n_{s}\right]+n_{0} \nabla_{\|} u_{s} \\
\frac{\partial}{\partial t}\left(u_{s}+\frac{q_{s}}{m_{s} c} A\right) & =-\frac{c}{B}\left[\phi, u_{s}+\frac{q_{s}}{m_{s} c} A\right]+\frac{T_{s}}{m_{s}} \nabla_{\|}\left(\frac{T_{\|_{s}}}{T_{s}}+\frac{n_{s}}{n_{0}}\right)-\frac{q_{s}}{m_{s}} \frac{\partial \phi}{\partial z},
\end{aligned}
$$




$$
\begin{aligned}
\frac{\partial T_{\|_{s}}}{\partial t} & =-\frac{c}{B}\left[\phi, T_{\|_{s}}\right]+\frac{2}{n_{0}} \nabla_{\|}\left(q_{\|_{s}}+n_{0} T_{s} u_{s}\right), \\
\frac{\partial q_{\|_{s}}}{\partial t} & =-\frac{c}{B}\left[\phi, q_{\|_{s}}\right]-v_{\mathrm{t}_{s}} \nabla_{\|}\left(\sqrt{6} \alpha_{s} q_{\perp_{s}}-\frac{3}{2} n_{0} v_{\mathrm{t}_{s}} T_{\|_{s}}\right), \\
\frac{\partial T_{\perp_{s}}}{\partial t} & =-\frac{c}{B}\left[\phi, T_{\perp_{s}}\right]+\frac{1}{n_{0}} \nabla_{\|} q_{\perp_{s}}, \\
\frac{\partial q_{\perp_{s}}}{\partial t} & =-\frac{c}{B}\left[\phi, q_{\perp_{s}}\right]-v_{\mathrm{t}_{s}} \nabla_{\|}\left(2 \sqrt{\frac{2}{3}} \alpha_{s} q_{\|_{s}}-n_{0} v_{\mathrm{t}_{s}} T_{\perp_{s}}\right),
\end{aligned}
$$

где $\nabla_{\|} f=(1 / B)[A, f]-\partial_{z} f ;$ этот оператор представляет собой нормированный градиент вдоль магнитного поля $\mathbf{B}=\nabla A \times \hat{z}+B \hat{z}$.

Уравнения (45) и (46) выражают закон сохранения числа частиц и канонических импульсов. Уравнения (47) и (48) определяют эволюцию флуктуаций температуры и тепловых потоков в направлении, параллельном постоянной компоненте магнитного поля, а уравнения (49) и (50) определяют аналогичную эволюцию флуктуаций, вызванных движением, перпендикулярным магнитному направляющему полю.

В любом динамическом поле имеет место перенос вещества со скоростью $\mathbf{E} \times \mathbf{B}$, что представлено первыми слагаемыми в правых частях каждого из уравнений, в которых появляется электростатический потенциал $\phi$. В дополнение к этому эффекту каждое поле эволюционирует в соответствии с градиентами вдоль поля для соседствующих моментов. Например, наличие градиентов скорости и тепловых потоков воздействует на эволюцию температуры. С другой стороны, параллельные (перпендикулярные) тепловые потоки подвергаются воздействию со стороны параллельных (соответственно перпендикулярных) градиентов температуры, а также со стороны перпендикулярных (соответственно параллельных) тепловых потоков. Это задается связями, содержащими коэффициенты $\alpha_{s}$.

Рассмотрев коэффициенты $\left(W_{4}^{45}\right)_{s},\left(W_{4}^{54}\right)_{s},\left(W_{5}^{44}\right)_{s},\left(W_{5}^{55}\right)_{s}$ из формул $(40)$, мы видим, что в гамильтоновой модели константы $\alpha_{s} \neq 0$. Следовательно, существование гамильтоновой структуры для модели со скобкой Пуассона вида (30) требует присутствия в уравнениях (25) и (27) членов, которые задают непосредственную связь между параллельными и перпендикулярными тепловыми потоками. Хотя, насколько мы знаем, гамильтонова структура модели в случае $\alpha_{s}=0$ неизвестна, тем не менее в ней энергия сохраняется.

\section{5. НОРМАЛЬНЫЕ ПОЛЯ И ИНВАРИАНТЫ КАЗИМИРА}

В этом разделе мы покажем, что существует альтернативный набор динамических переменных, в терминах которых модель (22)-(27) принимает более компактный вид, позволяющий обнаружить существование некоторых законов сохранения. В частности, скобка Пуассона в терминах этих новых переменных имеет удивительно простую форму, которая значительно облегчает нахождение инвариантов Казимира.

Чтобы определить новый подходящий набор динамических переменных, важно заметить, что общее уравнение для нашей модели может быть записано как

$$
\begin{aligned}
\frac{\partial g_{m_{s}}}{\partial t}= & -\frac{c}{B}\left[\phi, g_{m_{s}}\right]+\frac{v_{\mathrm{t}_{s}}}{B}\left(\mathcal{W}_{s}^{(1)}\right)^{m n}\left[A, g_{n_{s}}\right]-v_{\mathrm{t}_{s}}\left(\mathcal{W}_{s}^{(1)}\right)^{m n} \frac{\partial g_{n_{s}}}{\partial z}- \\
& -\delta_{m 1} v_{\mathrm{t}_{s}} \frac{q_{s}}{T_{s}} \frac{\partial \phi}{\partial z}+\sqrt{m !}\left(\delta_{m 0}+\delta_{m 2}\right) \frac{v_{\mathrm{t}_{s}}^{2}}{c} \frac{q_{s}}{T_{s}} \frac{\partial A}{\partial z}, \quad m=0,1, \ldots, 5 .
\end{aligned}
$$


Матрицы $\mathcal{W}_{s}^{(1)}$ являются действительными и симметричными, следовательно, каждая из таких матриц обладает шестью действительными собственными значениями $\lambda_{0 s}, \lambda_{1 s}, \ldots, \lambda_{5 s}$. Более того, для каждого типа $s$ существует такая ортогональная матрица $U_{s}$, что $U_{s}^{\mathrm{T}} \mathcal{W}_{s}^{(1)} U_{s}=\Lambda_{s}$, где $\Lambda_{s}=\operatorname{diag}\left(\lambda_{0 s}, \lambda_{1 s}, \ldots, \lambda_{5 s}\right)$. Если мы делаем замену переменных $\left(g_{0_{s}}, g_{1_{s}}, \ldots, g_{5_{s}}\right) \rightarrow\left(G_{0_{s}}, G_{1_{s}}, \ldots, G_{5_{s}}\right)$, где

$$
G_{i s}=\left(U_{s}^{\mathrm{T}}\right)^{i r} g_{r_{s}},
$$

то уравнения (51) преобразуются в

$$
\begin{aligned}
& \frac{\partial G_{i_{s}}}{\partial t}=-\frac{c}{B}\left[\phi-\lambda_{i s} \frac{v_{\mathrm{t}_{s}}}{c} A, G_{i s}\right]-v_{\mathrm{t}_{s}} \lambda_{i s} \frac{\partial G_{i_{s}}}{\partial z}- \\
& \quad-v_{\mathrm{t}_{s}} \sqrt{m !}\left(U_{s}^{\mathrm{T}}\right)^{i m}\left(\delta_{m 1} \frac{q_{s}}{T_{s}} \frac{\partial \phi}{\partial z}-v_{\mathrm{t}_{s}}\left(\delta_{m 0}+\delta_{m 2}\right) \frac{q_{s}}{T_{s}} \frac{\partial A}{\partial z}\right), \quad i=0,1, \ldots, 5 .
\end{aligned}
$$

Такая запись уравнений модели имеет некоторые преимущества по сравнению с изначальной формулировкой (51).

Действительно, уравнение эволюции для каждой переменной $G_{i s}$ зависит только от $G_{i s}$ (кроме неявной зависимости от остальных динамических переменных, возникающей из потенциалов $\phi$ и $A$ ), в то время как уравнения для $g_{i_{s}}$, вообще говоря, также содержат в явном виде динамические переменные $g_{(i-1)_{s}}$ и $g_{(i+1)}$. Также вид уравнения (53) в двумерном пределе, когда значением координаты $z$ можно пренебречь, показывает, что поля $G_{i s}$ являются лагранжевыми инвариантами. В двумерном пределе уравнения (53) могут быть записаны как

$$
\frac{\partial G_{i_{s}}}{\partial t}+\mathbf{v}_{i_{s}} \cdot \nabla G_{i_{s}}=0
$$

где вектор

$$
\mathbf{v}_{i_{s}}=\frac{c}{B} \hat{z} \times \nabla\left(\phi-\lambda_{i_{s}} \frac{v_{\mathrm{t}_{s}}}{c} A\right)
$$

описывает несжимаемое поле скоростей, переносящих динамическое поле $G_{i_{s}}$. Два вклада в $\mathbf{v}_{i s}$ связаны с полем скоростей $\mathbf{E} \times \mathbf{B}$ и со свободным полем, направленным вдоль "полоидального" магнитного поля в первоначальном дрейфовом кинетическом описании. Существование такого переносящего поля становится ясным, если модель сформулирована в терминах переменных $G_{i s}$.

Явные выражения для собственных значений $\lambda_{0 s}, \ldots, \lambda_{5 s}$ выписаны в приложении к настоящей статье. Столбцы $v_{s}$ матриц $U_{s}$ находятся из уравнения $\mathcal{W}_{s}^{(1)} v_{s}=\Lambda_{s} v_{s}$.

В целях дальнейшего анализа покажем, что первые три строки матриц $U_{s}$ имеют следующую структуру:

$$
U_{s}=\left(\begin{array}{cccc}
v_{(0)} & v_{(1)_{s}} & \cdots & v_{(5) s} \\
\lambda_{0 s} v_{(0) s} & \lambda_{1 s} v_{(1) s} & \cdots & \lambda_{5 s} v_{(5) s} \\
\frac{\lambda_{0 s}^{2}-1}{\sqrt{2}} v_{(0)_{s}} & \frac{\lambda_{1 s}^{2}-1}{\sqrt{2}} v_{(1)_{s}} & \cdots & \frac{\lambda_{5 s}^{2}-1}{\sqrt{2}} v_{(5) s} \\
\vdots & \vdots & \ddots & \vdots
\end{array}\right)
$$


где $v_{(0)}, v_{(1)_{s}}, \ldots, v_{(5)}-$ константы, определяющиеся условием нормировки. С учетом (56) уравнения (53) могут также быть записаны как

$$
\begin{array}{r}
\frac{\partial G_{i s}}{\partial t}=-\frac{c}{B}\left[\phi-\lambda_{i s} \frac{v_{\mathrm{t}_{s}}}{c} A, G_{i s}\right]-v_{\mathrm{t}_{s}} \lambda_{i s} \frac{\partial G_{i_{s}}}{\partial z}-\lambda_{i_{s}} v_{\mathrm{t}_{s}} v_{(i)} \frac{q_{s}}{T_{s}} \frac{\partial}{\partial z}\left(\phi-\lambda_{i_{s}} \frac{v_{\mathrm{t}_{s}}}{c} A\right) \\
i=0,1, \ldots, 5 .
\end{array}
$$

Гамильтонов функционал (29) в терминах новых переменных принимает вид

$$
\bar{H}\left(G_{01}, \ldots, G_{5 \mathcal{N}}\right)=\frac{1}{2} \sum_{s} \int d^{3} x\left[n_{0} T_{s} \sum_{i=0}^{5} G_{i s}^{2}+q_{s} n_{0} U_{s}^{0 l} G_{l_{s}} \phi-q_{s} n_{0} \frac{v_{\mathrm{t}_{s}}}{c} U_{s}^{1 l} G_{l_{s}} A\right]
$$

следовательно,

$$
\bar{H}_{i s}=n_{0} T_{s} G_{i_{s}}+q_{s} n_{0} v_{(i)_{s}}\left(\phi-\lambda_{i_{s}} \frac{v_{\mathrm{t}_{s}}}{c} A\right)
$$

Тот факт, что (57) имеет вид уравнения переноса, и выражение для производных функционала (59) дают основание предполагать, что в терминах новых переменных скобка Пуассона для нашей модели дается выражением

$$
\{F, G\}=\sum_{s} \sum_{i=0}^{5}\left[\frac{-c}{q_{s} B n_{0} v_{(i)}} \int d^{3} x G_{i_{s}}\left[\frac{\delta F}{\delta G_{i_{s}}}, \frac{\delta G}{\delta G_{i_{s}}}\right]-\lambda_{i_{s}} \frac{v_{\mathrm{t}_{s}}}{n_{0} T_{s}} \int d^{3} x \frac{\delta F}{\delta G_{i_{s}}} \frac{\partial}{\partial z} \frac{\delta G}{\delta G_{i_{s}}}\right]
$$

Можно непосредственно убедиться (например, используя метод из раздела 4), что скобка (60) является скобкой Пуассона и, в частности, удовлетворяет тождеству Якоби. Скобка (60) имеет структуру суммы линейных комбинаций скобок Пуассона, обозначенных индексами $i$ и $s$. Скобки Пуассона такого вида часто встречаются в редуцированных жидкостных моделях плазмы; примеры можно найти, например, в работах [28], [31], [38]. Непосредственно подставляя в уравнение $\partial_{t} G_{i_{s}}=\left\{G_{i_{s}}, \bar{H}\right\}$ скобку (60) и гамильтониан (58), убеждаемся, что уравнения модели (57) восстанавливаются.

Как было замечено выше, альтернативный набор переменных $G_{0 s}, G_{1 s}, \ldots, G_{5 s}$ ведет к значительному упрощению вида скобки Пуассона по сравнению со скобкой Пуассона из раздела 4 , записанной в переменных $g_{0 s}, g_{1 s}, \ldots, g_{5 s}$. Мы называем переменные, приводящие скобку Пуассона к ее простейшему виду, нормальными полями. Основное преимущество такого упрощения заключается в том, что из соотношения (60) можно непосредственно определить инварианты Казимира.

Напомним, что инвариант Казимира скобки Пуассона $\{\cdot, \cdot\}$ (см., например, работы [25], [26]) есть функционал $C$, удовлетворяющий условию

$$
\{C, F\}=0
$$

для любого функционала $F$. Так как инварианты Казимира коммутируют, в частности, с гамильтонианом, они также сохраняются при динамике системы. 
Из определения (61) очевидно, что для скобки (60) инварианты Казимира даются выражением

$$
C_{i_{s}}=\int d^{3} x G_{i_{s}}, \quad i=0,1, \ldots, 5
$$

Условие сохранения таких интегралов, таким образом, налагает ограничения на динамику модели. В частности, в двумерном пределе $z \rightarrow 0$ инварианты Казимира принимают вид

$$
C_{i s}=\int d^{3} x \mathcal{C}_{i s}\left(G_{i s}\right), \quad i=0,1, \ldots, 5,
$$

где $\mathcal{C}_{i s}$ - произвольные функции. Следовательно, при наличии такой пространственной инвариантности системы (что является разумным приближением для лабораторной и солнечной плазмы, где сильная компонента магнитного поля делает динамику не зависящей в главном порядке от одной координаты) получается, что эволюция модели ограничивается бесконечным числом законов сохранения. Они связаны с ролью полей $G_{i s}$ как лагранжевых инвариантов в двумерном пределе (см. раздел 4).

Заметим, что если $\alpha_{s}=0$ для данного типа $s$, то матрица $\mathcal{W}_{s}^{(1)}$ становится блочнодиагональной с собственными значениями

$$
\begin{array}{lll}
\lambda_{0 s}=1, & \lambda_{1 s}=-1, & \lambda_{2 s}=-\sqrt{3+\sqrt{6}} \\
\lambda_{3 s}=\sqrt{3+\sqrt{6}}, & \lambda_{4 s}=-\sqrt{3-\sqrt{6}}, & \lambda_{5 s}=\sqrt{3-\sqrt{6}}
\end{array}
$$

С другой стороны, соответствующая матрица $U_{s}$ записывается как

$$
\left(\begin{array}{cccccc}
0 & 0 & \frac{-3 \sqrt{3+\sqrt{6}}+\sqrt{6(3+\sqrt{6})}}{3 \sqrt{2}} & \frac{3 \sqrt{3+\sqrt{6}}-\sqrt{6(3+\sqrt{6})}}{3 \sqrt{2}} & \frac{3 \sqrt{3-\sqrt{6}}+\sqrt{6(3-\sqrt{6})}}{3 \sqrt{2}} & -3 \sqrt{3-\sqrt{6}}-\sqrt{6(3-\sqrt{6})} \\
0 & 0 & \frac{1}{\sqrt{2}} & \frac{1}{\sqrt{2}} & -\frac{1}{\sqrt{2}} & -\frac{1}{\sqrt{2}} \\
0 & 0 & -\sqrt{\frac{3+\sqrt{6}}{6}} & \sqrt{\frac{3+\sqrt{6}}{6}} & -\sqrt{\frac{3-\sqrt{6}}{6}} & \sqrt{\frac{3-\sqrt{6}}{6}} \\
0 & 0 & \frac{1}{\sqrt{2}} & \frac{1}{\sqrt{2}} & \frac{1}{\sqrt{2}} & \frac{1}{\sqrt{2}} \\
\frac{1}{\sqrt{2}}-\frac{1}{\sqrt{2}} & 0 & 0 & 0 & 0 \\
\frac{1}{\sqrt{2}} & \frac{1}{\sqrt{2}} & 0 & 0 & 0 & 0
\end{array}\right) .
$$

Ясно, что в этом случае $v_{(0)_{s}}=v_{(1)_{s}}=0$. Следовательно, скобка Пуассона (60) не существует, так как $v_{(0)}$ и $v_{(1)}$ дают нулевой знаменатель. Таким образом, несмотря на то что и при $\alpha_{s}=0$ система может быть записана в виде (57), гамильтонова формулировка модели с гамильтонианом (59) и скобкой Пуассона вида (60) невозможна; это подтверждает сделанные в разделе 4 выводы о случае $\alpha_{s}=0$.

\section{6. ЗАКЛЮЧЕНИЕ}

Мы представили гамильтонову анизотропную электромагнитную жидкостную модель с шестью полевыми переменными для каждого типа частиц, описывающую эволюцию плотности, канонического жидкостного импульса, а также флуктуации параллельных и перпендикулярных температур и тепловых потоков. Модель выражается через моменты (записывающиеся через полиномы Эрмита и Лагерра) 
обобщенной функции распределения и получается в результате замыкания системы уравнений с помощью условия пропорциональности момента $g_{40_{s}}$ (момента $g_{21_{s}}$ ) и флуктуаций перпендикулярного (соответственно параллельного) теплового потока. Такое замыкание означает введение членов, непосредственно связывающих эволюции параллельных и перпендикулярных тепловых потоков для каждого типа частиц.

В качестве гамильтониана жидкостной модели мы взяли сохраняющийся функционал, полученный самосогласованным образом из гамильтониана исходной дрейфовой кинетической модели. Затем мы построили скобку Пуассона, предполагая, что она имеет вид скобки Ли-Пуассона, как это принято в редуцированных моделях жидкой плазмы. Мы рассмотрели общий вид билинейного функционала, который удовлетворяет условию Лейбница и позволяет получить из взятого нами гамильтониана двумерные уравнения модели. С помощью наложения дополнительных ограничений, связанных с условием антисимметричности и тождеством Якоби, мы вывели выражение для скобки Ли-Пуассона модели в двумерном пределе. Расширение до трех измерений было проделано с помощью методики из работы [29]. Из выражения для скобки Пуассона вытекает, что коэффициенты пропорциональности $\alpha_{s}$ в соотношении замыкания не могут быть равны нулю, и это подразумевает наличие членов, связывающих параллельный и перпендикулярный тепловые потоки в уравнениях модели. Это гарантирует гамильтонову структуру со скобкой Ли-Пуассона.

Далее мы ввели альтернативный набор динамических переменных (нормальных полей), в терминах которых скобка Пуассона модели значительно упростилась. Это сделало определение инвариантов Казимира особенно простым. В двумерном пределе мы нашли $6 \mathcal{N}$ бесконечных семейств инвариантов Казимира. Они отражают тот факт, что нормальные поля являются лагранжевыми инвариантами модели. Тем самым обнаруживается замечательная особенность модели в двумерном пределе: она действительно может быть переформулирована как система $6 \mathcal{N}$ уравнений переноса для $6 \mathcal{N}$ лагранжевых инвариантов, перемещаемых несжимаемыми обобщенными полями скоростей. Такие поля скоростей, соответствующие полям скоростей в уравнениях (55), ассоциируются с функциями потоков, которые отвечают линейным комбинациям электростатического и магнитного потенциалов (скорости $\mathbf{E} \times \mathbf{B}$ и свободному потоку вдоль флуктуационной части магнитного поля). Подобная особенность встречается и в других редуцированных гамильтоновых моделях плазмы [31], [38], [28], [24], но ни одна из таких моделей не учитывает перпендикулярные температуру и тепловые потоки.

Наконец, следует сделать акцент на замечании о замыкании системы в случае $\alpha_{s}=0$. Это отвечает удалению членов, непосредственно связывающих параллельный и перпендикулярный тепловые потоки для данного типа частиц и соотносится с замыканием, которое было принято в работах [2], [7], где пренебрегалось влиянием конечного радиуса Лармора, неоднородностей фона и диссипации. Гамильтонова формулировка, полученная для нашей модели, не подходит к случаю $\alpha_{s}=0$. В этом случае энергия сохраняется, но из скобки Пуассона вида (30) мы не можем получить уравнения модели для принятого нами гамильтонова функционала. Это наводит на мысль о том, что для замыкания в случае $\alpha_{s}=0$ гамильтонова структура требует иного вида скобки Пуассона или что компоненты, которыми мы пренебрегали (например, эффекты, связанные с конечным радиусом Лармора), необходимы для восстановления гамильтоновой структуры с данным видом скобки Пуассона. 


\section{Собственные значения матриц $\mathcal{W}_{s}^{(1)}$}

\section{ПРИЛОЖЕНИЕ}

Для фиксированного типа частиц собственные значения матрицы $\mathcal{W}_{s}^{(1)}$ суть корни характеристического многочлена

$$
\lambda^{6}-\left(7+4 \alpha_{s}^{2}\right) \lambda^{4}+\left(9+12 \alpha_{s}^{2}\right) \lambda^{2}-3
$$

который мы можем записать как

$$
u^{3}-\left(7+4 \alpha_{s}^{2}\right) u^{2}+\left(9+12 \alpha_{s}^{2}\right) u-3, \quad u=\lambda^{2} .
$$

Следуя стандартной методике поиска корней кубического алгебраического уравнения, совершим преобразование $u=y+\left(7+4 \alpha_{s}^{2}\right) / 3$, которое придает уравнению для полинома (П.1) вид

$$
y^{3}-\frac{16 \alpha_{s}^{4}+20 \alpha_{s}^{2}+22}{3} y-\frac{32 \alpha_{s}^{2}+80 \alpha_{s}^{4}}{9}-\frac{128 \alpha_{s}^{6}+200}{27}=0 .
$$

Корни этого уравнения таковы:

$$
\begin{aligned}
y_{1}= & \frac{2}{3} \sqrt{2} \sqrt{8 \alpha_{s}^{4}+10 \alpha_{s}^{2}+11} \cos \left[\frac { 1 } { 3 } \operatorname { A r g } \left(\frac{4}{27}\left(16 \alpha_{s}^{6}+30 \alpha_{s}^{4}+12 \alpha_{s}^{2}+25\right)+\right.\right. \\
& \left.\left.+\frac{2}{3} i \sqrt{2} \sqrt{24 \alpha_{s}^{8}+40 \alpha_{s}^{6}+36 \alpha_{s}^{4}+30 \alpha_{s}^{2}+1}\right)\right], \\
y_{2}= & \frac{2}{3} \sqrt{2} \sqrt{8 \alpha_{s}^{4}+10 \alpha_{s}^{2}+11} \cos \left[\frac { 1 } { 3 } \operatorname { A r g } \left(\frac{4}{27}\left(16 \alpha_{s}^{6}+30 \alpha_{s}^{4}+12 \alpha_{s}^{2}+25\right)+\right.\right. \\
& \left.\left.+\frac{2}{3} i \sqrt{2} \sqrt{24 \alpha_{s}^{8}+40 \alpha_{s}^{6}+36 \alpha_{s}^{4}+30 \alpha_{s}^{2}+1}+2 \pi\right)\right], \\
y_{3}= & \frac{2}{3} \sqrt{2} \sqrt{8 \alpha_{s}^{4}+10 \alpha_{s}^{2}+11} \cos \left[\frac { 1 } { 3 } \operatorname { A r g } \left(\frac{4}{27}\left(16 \alpha_{s}^{6}+30 \alpha_{s}^{4}+12 \alpha_{s}^{2}+25\right)+\right.\right. \\
& \left.\left.\left.+\frac{2}{3} i \sqrt{2} \sqrt{24 \alpha_{s}^{8}+40 \alpha_{s}^{6}+36 \alpha_{s}^{4}+30 \alpha_{s}^{2}+1}\right)+4 \pi\right)\right] .
\end{aligned}
$$

Таким образом, собственные значения матрицы $\mathcal{W}_{s}^{(1)}$ записываются как

$$
\lambda_{0,1_{s}}= \pm \sqrt{y_{1}+\frac{7+4 \alpha_{s}^{2}}{3}}, \quad \lambda_{2,3_{s}}= \pm \sqrt{y_{2}+\frac{7+4 \alpha_{s}^{2}}{3}}, \quad \lambda_{4,5_{s}}= \pm \sqrt{y_{3}+\frac{7+4 \alpha_{s}^{2}}{3}} .
$$

Благодарности. Автор выражает свою благодарность за полезные дискуссии Nonlinear Dynamics Team (Centre de Physique Théorique).

\section{Список литературы}

[1] A. Brizard, Phys. Fluids B, 4:5 (1992), 1213-1228.

[2] B. Scott, Phys. Plasmas, 17:10 (2010), 102306.

[3] T. Passot, P. L. Sulem, Phys. Plasmas, 11:11 (2004), 5173-5189.

[4] T. Passot, P. L. Sulem, Phys. Plasmas, 14:8 (2007), 082502, 14 pp.

[5] T. Passot, P. L. Sulem, P. Hunana, Phys. Plasmas, 19:8 (2012), 082113. 
[6] P. B. Snyder, G. W. Hammett, W. Dorland, Phys. Plasmas, 4:11 (1997), 3974-3985.

[7] P. B. Snyder, G. W. Hammett, Phys. Plasmas, 8:7 (2001), 3199-3216.

[8] H. Sugama, T.-H. Watanabe, W. Horton, Phys. Plasmas, 8:6 (2001), 2617-2628.

[9] Y. Sarazin, G. Dif-Pradalier, D. Zarzoso, X. Garbet, Ph. Ghendrih, V. Grandgirard, Plasma Phys. Control. Fusion, 51:11 (2009), 115003, 14 pp.

[10] P. J. Morrison, Phys. Lett. A, 80:5-6 (1980), 383-386.

[11] J. E. Marsden, A. Weinstein, Phys. D, 4:3 (1982), 394-406.

[12] Б. А. Купершмидт, Ю. И. Манин, Функи. анализ и его прил., 12:1 (1978), 25-37.

[13] J. Gibbons, Phys. D, 3:3 (1981), 503-511.

[14] J. Gibbons, D. D. Holm, C. Tronci, Phys. Lett. A, 372:7 (2008), 1024-1033.

[15] J. E. Marsden, P. J. Morrison, A. Weinstein, "The Hamiltonian structure of the BBGKY hierarchy equations", Fluids and Plasmas: Geometry and Dynamics (University of Colorado, Boulder, Colorado, USA, July 17-23, 1983), Contemporary Mathematics, 28, ed. J. E. Marsden, AMS, Providence, RI, 1984, 115-124.

[16] A. A. Chesnokov, M. V. Pavlov, Acta Appl. Math., 122:1 (2012), 367-380.

[17] M. Perin, C. Chandre, P. J. Morrison, E. Tassi, Ann. Phys., 348 (2014), 50-63.

[18] M. Perin, C. Chandre, P. J. Morrison, E. Tassi, J. Phys. A: Math. Theor., 48:27 (2015), 275501, 24 pp.

[19] E. Tassi, J. Phys. A: Math. Theor., 47:19 (2014), 195501, 25 pp.

[20] E. Tassi, Eur. Phys. J. D, 68:7 (2014), 196-206.

[21] E. Tassi, J. Phys.: Conf. Ser., 561:1 (2014), 012018, 9 pp.

[22] E. Tassi, Ann. Phys., 362 (2015), 239-260.

[23] R. D. Hazeltine, J. D. Meiss, Plasma Confinement, Dover, New York, 2003.

[24] D. Grasso, E. Tassi, J. Plasma Phys., 81:5 (2015), 495810501, 22 pp.

[25] P. J. Morrison, Rev. Modern Phys., 70:2 (1998), 467-522.

[26] J.E. Marsden, T.S. Ratiu, Introduction to Mechanics and Symmetry. A Basic Exposition of Classical Mechanical Systems, Texts in Applied Mathematics, 17, Springer, New York, 2010.

[27] H. J. de Blank, "Kinetic electrons in drift-Alfv'en current-vortex filaments", Proceedings of the 28th Conference on Controlled Fusion and Plasma Physics (Funchal, Madeira, Portugal, 18-22 June, 2001), Europhysics Conference Abstracts (ECA), 25A, EPS, France, 2001, $1709-1712$.

[28] F. L. Waelbroeck, E. Tassi, Commun. Nonlinear Sci. Numer. Simul., 17:5 (2012), 2171-2178.

[29] E. Tassi, P. J. Morrison, D. Grasso, F. Pegoraro, Nucl. Fusion, 50:3 (2010), 034007, 8 pp.

[30] E. Tassi, P. J. Morrison, F. L. Waelbroeck, D. Grasso, Plasma Phys. Control. Fusion, 50:8 (2008), 085014, 29 pp.

[31] F. L. Waelbroeck, R. D. Hazeltine, P. J. Morrison, Phys. Plasmas, 16:3 (2009), 032109.

[32] F. L. Waelbroeck, P. J. Morrison, W. Horton, Plasma Phys. Control. Fusion, 46:9 (2004), 1331-135.

[33] R. D. Hazeltine, C. T. Hsu, P. J. Morrison, Phys. Fluids, 30:10 (1987), 3204-3211.

[34] D. Dagnelund, V. P. Pavlenko, Phys. Scr., 71:3 (2005), 293-297.

[35] A. Weinstein, Phys. Fluids, 26:2 (1983), 388-390.

[36] B. N. Kuvshinov, F. Pegoraro, T. J. Schep, Phys. Lett. A, 191:3-4 (1994), 296-300.

[37] J.-L. Thiffeault, P. J. Morrison, Phys. D, 136:3-4 (2000), 205-244.

[38] E. Cafaro, D. Grasso, F. Pegoraro, F. Porcelli, A. Saluzzi, Phys. Rev. Lett., 80:20 (1998), 4430-4434. 